\title{
Molecular genetic overlap between migraine and major depressive disorder
}

\author{
Yuanhao Yang ${ }^{1,2}$. Huiying Zhao ${ }^{1,3} \cdot$ Dorret I Boomsma $^{4}$ Lannie Ligthart ${ }^{4}$ Andrea C. Belin ${ }^{5} \cdot$ George Davey Smith $^{6}$.

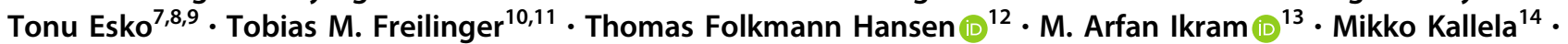 \\ Christian Kubisch ${ }^{15}$. Christofidou Paraskevi ${ }^{16}$. David P. Strachan $\left(10^{17} \cdot\right.$ Maija Wessman ${ }^{18,19} \cdot$ The International \\ Headache Genetics Consortium - Arn M. J. M. van den Maagdenberg ${ }^{20,21} \cdot$ Gisela M. Terwindt $^{20} \cdot$ Dale R. Nyholt $^{1}$
}

Received: 24 October 2017 / Accepted: 23 March 2018 / Published online: 11 July 2018

(c) European Society of Human Genetics 2018

\begin{abstract}
Migraine and major depressive disorder (MDD) are common brain disorders that frequently co-occur. Despite epidemiological evidence that migraine and MDD share a genetic basis, their overlap at the molecular genetic level has not been thoroughly investigated. Using single-nucleotide polymorphism (SNP) and gene-based analysis of genomewide association study (GWAS) genotype data, we found significant genetic overlap across the two disorders. LD Score regression revealed a significant SNP-based heritability for both migraine $\left(h^{2}=12 \%\right)$ and MDD $\left(h^{2}=19 \%\right)$, and a significant cross-disorder genetic correlation $\left(r_{\mathrm{G}}=0.25 ; P=0.04\right)$. Meta-analysis of results for 8,045,569 SNPs from a migraine GWAS (comprising 30,465 migraine cases and 143,147 control samples) and the top 10,000 SNPs from a MDD GWAS (comprising 75,607 MDD cases and 231,747 healthy controls), implicated three SNPs (rs146377178, rs672931, and rs11858956) with novel genome-wide significant association $\left(P_{\mathrm{SNP}} \leq 5 \times 10^{-8}\right)$ to migraine and MDD. Moreover, gene-based association analyses revealed significant enrichment of genes nominally associated $\left(P_{\text {gene-based }} \leq\right.$ $0.05)$ with both migraine and MDD $\left(P_{\text {binomial-test }}=0.001\right)$. Combining results across migraine and MDD, two genes, $A N K D D 1 B$ and $K C N K 5$, produced Fisher's combined gene-based $P$ values that surpassed the genome-wide significance threshold $\left(P_{\text {Fisher's-combined }} \leq 3.6 \times 10^{-6}\right)$. Pathway analysis of genes with $P_{\text {Fisher's-combined }} \leq 1 \times 10^{-3}$ suggested several pathways, foremost neural-related pathways of signalling and ion channel regulation, to be involved in migraine and MDD aetiology. In conclusion, our study provides strong molecular genetic support for shared genetically determined biological mechanisms underlying migraine and MDD.
\end{abstract}

These authors contributed equally: Arn M.J.M. van den Maagdenberg, Gisela M. Terwindt, Dale R. Nyholt.

Members of the consortium are listed at the end of the paper.

Electronic supplementary material The online version of this article (https://doi.org/10.1038/s41431-018-0150-2) contains supplementary material, which is available to authorized users.

Yuanhao Yang y.yang@imb.uq.edu.au

$\triangle$ Dale R. Nyholt d.nyholt@qut.edu.au

Extended author information available on the last page of the article

\section{Introduction}

Migraine and major depressive disorder (MDD) are among the most common neurological disorders, each affecting $\sim 10-20 \%$ of the population with more females than males affected [1-3]. Bidirectional comorbidity between migraine and MDD is widely recognized [4-8]; it highly reduces the quality of life of patients with a huge impact on relatives and society [9]. As epidemiological studies have revealed a moderate heritability for migraine and MDD with estimates ranging from 30-50\% [10, 11], shared genetic factors may underlie these disorders [12-14], although molecular evidence for such an association is lacking.

A number of genome-wide association studies (GWAS) have been conducted separately for migraine [15-21] and MDD [22-33]. For both disorders an 
increasing number of loci have been identified. The largest, latest, migraine GWAS analysed 59,674 cases and 316,078 healthy controls and identified 38 genome-wide significant $\left(P_{\mathrm{SNP}} \leq 5 \times 10^{-8}\right)$ loci containing 44 independent single nucleotide polymorphisms (SNPs) associated with migraine risk [21]. While the largest, latest, MDD GWAS analysed a combined 130,620 self-reported and clinically evaluated lifetime major depression cases and 347,620 controls identified 15 genome-wide significant loci, containing 17 independent SNPs associated with MDD risk [33]. Comparison of the genome-wide significant loci between the two disorders yielded no shared loci. It remains however of interest to investigate whether signals of other SNPs, below the threshold for genomewide significance, reveal molecular genetic overlap between migraine and MDD.

Compared to epidemiological studies, analyzing GWAS SNP data provides an opportunity to test for genetic overlap between migraine and MDD at the molecular genetic level and can yield genetic risk variants associated with both migraine and MDD. Also, extending the genetic overlap analysis from the SNP-level to the gene-level-given that genes are the predominant functional unit of the human genome and more closely related to biology than individual SNPs-can (i) provide novel evidence on the genetic association between migraine and MDD; (ii) give insight into shared biological pathways underlying the two disorders; and (iii) help identify target genes for drug development. Moreover, the identification of genetic overlap and specific genetic variants shared across disorders can be used to assess the validity of the clinical diagnosis and classification of patients.

Here we examined the genetic overlap across migraine and MDD by (i) evaluating SNP-based genetic overlap utilizing LD (linkage disequilibrium) Score regression (LDSC) and SNP effect concordance analysis (SECA) using genome-wide summary statistics from the 2016 International Headache Genetics Consortium (IHGC) migraine and 2013 Psychiatric Genomics Consortium (PGC) MDD GWAS, and the 'top' 10,000 most significant SNP results from the 2016 23andMe MDD GWAS; (ii) identifying genetic risk variants associated with both migraine and MDD by meta-analysis of 2016 IHGC migraine and 2016 23andMe MDD GWAS results; (iii) evaluating gene-level genetic overlap across migraine and MDD to identify genes associated with migraine and MDD using gene-based association analysis of summary statistics from the 2016 IHGC migraine and 2013 PGC MDD GWAS; and (iv) exploring the biological pathways represented by the genes showing association to migraine and MDD.

\section{Materials and methods}

\section{Study samples}

\section{IHGC migraine GWAS}

The 2016 IHGC (http://www.headachegenetics.org/) migraine GWAS sample comprised 59,674 migraine cases and 316,078 healthy controls [21]; all participants were of European ancestry. Migraine phenotypes were diagnosed by self-reported questionnaires or clinical interviews according to the International Classification of Headache Disorders (ICHD) criteria [34]. Subjects in each individual GWAS had their specific standard genotyping platform and quality control criteria, which were summarized elsewhere [21]; all subjects were imputed using the 1000 Genomes Project [35] reference panel (Phase I, v3 release or later). Each individual GWAS also performed their association analysis independently, adjusted for sex and the top ten principal components to account for potential population stratification where required [21]. A combined fixed-effect (FE) metaanalysis was then performed using the Genome-wide Association Meta-Analysis (GWAMA) program [36]. After SNP filtering, the final 2016 IHGC migraine GWAS included association results for 8,045,569 SNPs. For more detailed descriptions of the migraine cohorts and statistical analyses, please refer to the original publication [21]. Here, we utilized the GWAMA output after excluding results from the 23andMe GWAS sample (30,465 migraine cases and 143,147 controls), leaving a total migraine GWAS sample of 29,209 cases and 172,931 controls, to ensure there was no sample overlap between 2016 IHGC migraine GWAS and the 2016 23andMe MDD GWAS.

\section{PGC MDD GWAS}

The 2013 PGC (http://pgc.unc.edu) MDD GWAS sample comprised 18,759 unrelated participants of European ancestry (9240 MDD cases and 9519 healthy controls) from nine MDD GWA case-control samples. [23] All MDD cases were diagnosed by a structured clinical interview or clinical-based checklist according to the Diagnostic and Statistical Manual of Mental Disorders, fourth edition (DSM-IV) criteria. [37] The PGC performed a mega-analysis, which required centralizing the genotype data from all GWA samples prior to performing consistent QC, imputation and association analysis. Individual genotypes were all imputed up to the CEU (Utah Residents with Northern and Western European Ancestry) and TSI (Toscani in Italy) HapMap3 reference panel. Association analysis was carried out using a logistic regression assuming an additive SNP effect (allelic 
association) model. The final 2013 PGC MDD GWAS comprised results for $1,232,794$ SNPs.

\section{23andMe MDD GWAS}

The 2016 23andMe MDD discovery GWAS sample [33] recruited 307,354 subjects of European ancestry, including 75,607 self-reported MDD cases and 231,747 healthy controls. Subjects were systematically genotyped, QCd, and imputed using the 1000 Genomes Project [35] Phase I reference panel. Under the assumption of additive allelic effects, GWA analysis was performed using logistic regression adjusted for age, sex, and the top five ancestry principal components. After removing SNPs with low quality imputation, 13,519,496 SNPs were included in the discovery GWAS; and only the top 10,000 most significant SNPs (http://www.nature.com/ng/journal/v48/n9/full/ng. 3623.html\#supplementary-information) with $P \leq 1 \times 10^{-5}$ were available for download and used in our study.

In addition to the above details and original publications describing the GWAS summary statistics analysed in our study-including URLs from where the data can be obtained online-details and data from the analysed datasets are available from the GWAS Central database (http://www.gwascentral.org/study/HGVST1855).

\section{Genetic analyses}

\section{LD score regression to evaluate genetic similarity}

LD scores were calculated according to the European 1000 Genomes Project haplotype reference data (Phase I, v3). LD score regression was performed using the LDSC software (https://github.com/bulik/ldsc) [38]. GWAS summary statistics from the 2016 IHGC migraine GWAS and the 2013 PGC MDD GWAS were utilized in this analysis. GWAS data were reformatted and harmonized utilizing the "munge_sumstats.py" script, based on the SNP list used in LD score calculation. As per the LDSC manual, SNPs were removed if they were not present in the relevant reference data, had a rare frequency (minor allele frequency $[\mathrm{MAF}] \leq 0.01$ ), were poorly imputed (INFO score $\leq 0.90$ ) or strand-ambiguous. We first performed single-trait $\mathrm{LD}$ Score regression to evaluate the SNP-based liability heritability $\left(h_{\text {SNP }}^{2}\right)$ for the 2016 IHGC migraine and 2013 PGC MDD GWAS, using their sample prevalence of $14.5 \%$ for migraine and $49.3 \%$ for MDD, and a population prevalence of $15 \%$ for both migraine and MDD; and then built a cross-trait LD Score regression to estimate the genetic correlation $\left(r_{\mathrm{G}}\right)$ between migraine and MDD.

\section{SECA analysis to evaluate genetic overlap}

Whereas LD Score regression requires GWAS results for millions of SNPs spread evenly across the genome, SECA is able to assess genetic overlap for a subset of SNPs. [39] Since only the top 10,000 most significant SNPs ( $P$ value $\leq$ $1 \times 10^{-5}$ ) were available for the 201623 andMe MDD GWAS, we utilized SECA to examine the genetic overlap between the 2016 IHGC migraine GWAS and 2016 23andMe MDD GWAS. SECA first aligned the SNP effects across the two GWA study summary results to the same effect allele, and then extracted a subset of independent SNPs via ' $P$ value informed' linkage disequilibrium (LD) clumping. The approach iterated from the first to last SNP on each chromosome sorted from smallest to largest 2016 IHGC migraine GWAS $P$ value that had not already been clumped (denoting this as the 'index' SNP) and formed clumps of all other SNPs that are within $1 \mathrm{Mb}$ and in $\mathrm{LD}\left(r^{2}\right.$ $>0.1$, based on $1000 \mathrm{G}$ PhaseI v3 CEU genotype data) with the index SNP. A second round of LD clumping was performed to clump any of the round 1 index SNPs within 10 $\mathrm{Mb}$ of each other to account for long-range $\mathrm{LD}\left(r^{2}>0.1\right)$. The approach identified the subset of independent (index) SNPs with the most significant association $P$ values in the 2016 IHGC migraine GWAS. After subgrouping SNPs with $P$ value thresholds $P \leq\{0.01,0.05,0.1,0.2,0.3,0.4,0.5$, $0.6,0.7,0.8,0.9,1.0\}$ in the 2016 IHGC migraine GWAS, two-sided exact binomial tests were performed to assess the concordance of SNP effect directions across the 2016 IHGC migraine GWAS and 2016 23andMe MDD GWAS results.

\section{Meta-analysis of migraine and MDD}

Meta-analysis of the 2016 IHGC migraine GWAS and 2016 23andMe MDD GWAS (for the top 10,000 SNPs) was performed using the METASOFT (URL: http://genetics.cs. ucla.edu/meta/) inverse variance-weighted FE model, [40] where the SNP effect size estimates $(\beta)$ are weighted by their estimated standard errors $(s e)$ to calculate a metaanalysis $P$ value. To allow for the presence of effect heterogeneity across studies, the METASOFT Han and Eskin's random-effects (RE2) model [40] was also utilized, as it is optimised to detect associations under heterogeneity. A total of 8687 SNPs in both the 2016 IHGC migraine and 2016 23andMe MDD GWAS were included in the meta-analysis. Following the meta-analysis, we calculated the LD $\left(r^{2}\right)$ between the implicated SNPs $\left(P_{\mathrm{SNP}} \leq 5 \times 10^{-8}\right)$ at each locus using PLINK (http://pngu.mgh.harvard.edu/purcell/ plink/) [41] and the European 1000 Genomes Project haplotype reference data (Phase I, v3). 


\section{Gene-based analysis to evaluate genetic overlap}

A gene-based approach [42] was also used to evaluate the genetic overlap across the 2016 IHGC migraine GWAS and 2013 PGC MDD GWAS. After downloading RefSeq gene information (hg19) from the UCSC genome browser (accessed 20 March 2014), overlapping isoforms of the same gene were combined to form a single full-length version of the gene, while isoforms that did not overlap were left as duplicates of that gene. This led to 23,438 unique genes. The common SNPs from the 2016 IHGC migraine and 2013 PGC MDD GWAS were then assigned to genes if they mapped to between $15 \mathrm{~kb} 5^{\prime}$ of the transcription start site (TSS) and $15 \mathrm{~kb} \mathrm{3}$ 'of the transcription end site (TES). This $15-\mathrm{kb}$ gene boundary extension was chosen based on the observation that $90 \%$ of SNPs effecting expression quantitative trait loci (eQTLs) are within this proximity [43]. Gene-based association tests were performed using the GATES test [44] implemented in the Fast ASsociation Tests (FAST) package [45]. GATES performs gene-based tests by adjusting the observed $P$ value of the most significant SNP assigned to a gene by the total effective number of independent SNPs tested across the gene. GATES performs eigenvalue analysis of the $n \times n$ SNP correlation matrix (estimated from the 1000 Genomes Project [released on May 2012] CEU reference population) for each gene to estimate the effective number of independent SNPs. The original report introducing the GATES gene-based test used computer simulation to demonstrate that the test offers effective control of the type 1 error rate regardless of gene size and LD pattern among SNPs, and does not need permutation or simulation to validate significance [44].

Given that gene-based association results may be correlated across neighbouring genes due to LD between the most significant SNP assigned to each gene, we estimated the effective number of independent genes (i.e., number of independent gene-based tests) by examining the LD between the top (most) significant SNP assigned to each gene. This calculation was performed using the Genetic type I Error Calculator (GEC) [46]. The GEC approach first divides the input SNPs into LD blocks, and assumes LD blocks are independent by ensuring the SNPs between blocks are not in LD $\left(r^{2}<0.1\right)$. GEC subsequently performs eigenvalue analysis of the correlation matrix for each LD block to estimate the effective number of independent SNPs. In the original report introducing the GEC [46], computer simulation and permutation was used to demonstrate that using the GEC estimate of the effective number of independent SNPs in a Bonferroni procedure yields correct type I error rates and behaves similarly to the gold standard of permutation.

To test genetic overlap, we first generated gene sets for each disorder based on three levels of significance (i.e., gene-based $P$ value $\leq 0.01,0.05$, or 0.1 ) to allow for differences in power across the different GWA studies, and then calculated the effective number of independent genes per disorder. Next, we set the 2016 IHGC migraine GWAS as the 'discovery' dataset and 2013 PGC MDD GWAS as the 'target' dataset to test for genetic overlap according to three $P$ value significance levels (e.g., test whether the proportion of genes with a gene-based $P$ value $\leq 0.05$ for both migraine and MDD was more than expected by chance). The observed number of overlapping genes was defined as the effective number of genes with $P$ values less than the threshold in both the discovery and target datasets. The observed proportion of overlapping genes was the observed effective number of overlapping genes divided by the effective number of genes with a $P$ value less than the threshold in the discovery dataset. The expected proportion of overlapping genes was the effective number of genes with a $P$ value less than the threshold in the target dataset divided by the total effective number of genes in the target dataset. The statistical significance of whether the number of overlapping genes was more than expected by chance was calculated using one-sided exact binomial tests. Moreover, to identify the individual genes associated across migraine and MDD, we combined gene-based evidence for association across the two disorders using the Fisher's combined $P$ value approach. This gene-based approach was recently utilized to show gene-based pleiotropy across migraine with aura and migraine without aura [42], as well as the five major disorders in the PGC: attention deficit hyperactivity disorder, autism spectrum disorder, bipolar disorder, MDD, and schizophrenia [47].

\section{Pathway analysis of overlapping genes}

To discover shared biological pathways underlying migraine and MDD, we performed a pathway analysis of the significant overlapping genes from the gene-based analysis using the $\mathrm{g}$ :GOSt tool of the $\mathrm{g}$ :Profiler web server (http://biit.cs.ut.ee/gprofiler/) [48]. The overlapping genes with Fisher's combined $P$ value lower than $1 \times 10^{-3}$ were selected [49] and evaluated using the g:Profiler web server. The g:GOSt tool can identify significantly enriched pathways through different functional databases including Gene Ontology (GO) [50] (biological process, cellular component and molecular function), Kyoto Encyclopedia of Genes and Genomes (KEGG) [51], and Reactome [52]. Further advanced options are also available for term filtering, including the functional category size thresholds for limiting enrichment analyses and the significance threshold for multiple testing (e.g., Benjamini-Hochberg False Discovery Rate [FDR], and Bonferroni correction). For our analyses, no size boundaries were set for functional category and term intersection; Benjamini-Hochberg FDR was utilized for multiple testing correction; and other advanced options 
Table 1 Summary of LD score regression

\begin{tabular}{|c|c|c|c|c|c|}
\hline \multicolumn{6}{|c|}{ SNP-based heritability } \\
\hline $\begin{array}{l}\text { Pheno- } \\
\text { type }\end{array}$ & \multicolumn{2}{|c|}{ Number of valid SNPs in analysis } & \multicolumn{2}{|c|}{$h_{\mathrm{SNP}}^{2}(95 \% \mathrm{CI})$} & Estimated intercept (se) \\
\hline $\begin{array}{l}2016 \\
\text { IHGC } \\
\text { migrai- } \\
\text { ne }\end{array}$ & \multicolumn{2}{|l|}{834,361} & \multicolumn{2}{|c|}{$12.31 \%(9.17-15.45 \%)$} & $1.08(0.015)$ \\
\hline $\begin{array}{l}2013 \\
\text { PGC } \\
\text { MDD }\end{array}$ & \multicolumn{2}{|l|}{877,768} & \multicolumn{2}{|c|}{$19.10 \%(12.16-26.04 \%)$} & $1.01(0.0083)$ \\
\hline \multicolumn{6}{|c|}{ SNP-based genetic correlation } \\
\hline Phenotype 1 & Phenotype 2 & \multicolumn{2}{|c|}{$\begin{array}{l}\text { Number of valid } \\
\text { SNPs in analysis }\end{array}$} & $\begin{array}{l}r_{G}(s e)[P \\
\text { value }]\end{array}$ & $\begin{array}{l}\text { Estimated intercept for } \\
\text { genetic covariance }(\mathrm{se})\end{array}$ \\
\hline $\begin{array}{l}2016 \text { IHGC } \\
\text { migraine }\end{array}$ & 2013 PGC MDD & 686,287 & & $\begin{array}{l}0.25(0.12) \\
{[0.040]}\end{array}$ & $0.040(0.0075)$ \\
\hline
\end{tabular}

$h_{S N P}^{2}$ SNP-based heritability, 95\% CI 95\% confidence interval, $r_{G}$ SNP-based genetic correlation, se standard error were kept as their default. Analyses were first run without including then run including electronic GO annotations. Lastly, given such pathway analyses may be biased in the presence of strong LD across neighbouring genes, we ensured the enriched pathways did not contain genes with top significant SNPs in LD $\left(r^{2}>0.1\right)$.

\section{Results}

\section{LD score-based genetic correlation between migraine and MDD}

As summarized in Table 1, using LD score regression with no intercept constraining, we observed a significant SNPbased liability-scale heritability of $12 \%$ (95\% CI: 9-15\%) for 2016 IHGC migraine, and 19\% (95\% CI: 12-26\%) for 2013 PGC MDD. For cross-trait analysis, a significant positive genetic correlation $\left(r_{\mathrm{G}}\right)$ of $0.25 \quad(95 \%$ CI: $0.01-0.48$ ) was estimated between the 2016 IHGC migraine and 2013 PGC MDD GWAS.

\section{SECA-based genetic concordance between migraine and MDD}

SECA revealed a significant genetic concordance between genetic risk factors (SNP risk alleles) for 2016 IHGC migraine and 2016 23andMe MDD. For instance, the SNP effect concordance between migraine and MDD is considerable given that of the 358 independent SNPs with the smallest $P$ values in the 2016 IHGC migraine GWAS, the risk increasing allele for MDD and migraine was the same for $202(56.42 \%$, two-sided binomial test $P=0.017)$.
The SNP effect concordance was further enriched (by 31\%) in the subset of independent SNPs with nominal MDD association $(P \leq 0.05)$, with $34(73.91 \%)$ out of 46 independent SNPs having the same risk increasing allele for migraine and MDD (two-sided binomial test $P=0.0016$ ). SNP effect concordance results for all 12 analysed $P$ value thresholds are provided in Table 2.

\section{Genetic risk variants associated with both migraine and MDD}

A total of 683,106 participants were included in the metaanalysis of 2016 IHGC migraine and 2016 23andMe MDD GWAS. In total, 542 SNPs at 9 genomic loci produced evidence for genome-wide significant association $\left(P_{\mathrm{SNP}} \leq\right.$ $5 \times 10^{-8}$ ) based on the FE model (Supplementary Table 1 contains meta-analysis results for the 542 SNPs using both the FE and RE2 model). After examining LD between the most significant (top), or "index", risk SNPs, 9 independent SNPs were identified (Table 3).

Among these nine independent SNPs, five (rs12127789 hg19.chr1:g.72740073G>T, rs2195636 hg19.chr3: g.158352440C $>$ T, rs768705 hg19.chr5:g.87568710A $>$ G, rs9536359 hg19.chr13:g.53691446C $>\mathrm{T}$ and rs5751069 hg19.chr22:g.41627775C $>$ G) presented a significantly stronger association with MDD (either the SNP showed a genome-wide significant association or the SNP was in LD with a genome-wide significant SNP) compared to migraine, indicating that these SNPs are predominantly driven by the association signal in the 201623 andMe MDD GWAS. However, one SNP (rs6476606 hg19.chr9: g. 37005561 $\mathrm{A}>\mathrm{G})$ showed near-suggestive association $\left(P_{\mathrm{SNP}} \leq 1 \times 10^{-5}\right)$ with both $\mathrm{MDD}\left(P_{\mathrm{SNP}}=1.5 \times 10^{-5}\right)$ and 
Table 2 SECA-based genetic concordance between migraine and MDD

\begin{tabular}{llllll}
\hline $\begin{array}{l}\text { 2016 IHGC migraine } \\
\begin{array}{l}\text { GWAS } P \text { value } \\
\text { threshold }\end{array}\end{array}$ & $\begin{array}{l}\text { No. of SNP effects across } \\
\text { migraine and MDD }\end{array}$ & & $\begin{array}{l}\text { Proportion of SNPs with } \\
\text { concordant effects }\end{array}$ & $\begin{array}{l}\text { Concordance test } P \\
\text { value }^{\mathrm{b}}\end{array}$ \\
\cline { 2 - 5 } & Concordant & Discordant & Total & \\
\hline 1 & 202 & 156 & 358 & 0.5642 & $1.73 \times 10^{-2}$ \\
0.9 & 192 & 150 & 342 & 0.5614 & $2.65 \times 10^{-2}$ \\
0.8 & 178 & 140 & 318 & 0.5597 & $3.78 \times 10^{-2}$ \\
0.7 & 166 & 124 & 290 & 0.5724 & $1.59 \times 10^{-2}$ \\
0.6 & 159 & 112 & 271 & 0.5867 & $5.11 \times 10^{-3}$ \\
0.5 & 146 & 100 & 246 & 0.5935 & $4.03 \times 10^{-3}$ \\
0.4 & 126 & 85 & 211 & 0.5972 & $5.76 \times 10^{-3}$ \\
0.3 & 109 & 69 & 178 & 0.6124 & $3.35 \times 10^{-3}$ \\
0.2 & 88 & 46 & 134 & 0.6567 & $3.60 \times 10^{-4}$ \\
0.1 & 66 & 22 & 88 & 0.7500 & $2.88 \times 10^{-6}$ \\
0.05 & 34 & 12 & 46 & 0.7391 & $1.64 \times 10^{-3}$ \\
0.01 & 14 & 3 & 17 & 0.8235 & $1.27 \times 10^{-2}$ \\
\hline
\end{tabular}

${ }^{a}$ There were a total of 358 independent SNPs (linkage disequilibrium $r^{2}<0.1$ ) with results in both the 2016 IHGC Migraine GWAS and the top 10,000 SNPs $\left(P\right.$ value $\left.\leq 1 \times 10^{-5}\right)$ in the 201623 andMe MDD GWAS

${ }^{\mathrm{b}}$ Two-sided exact binomial test for concordance: where the number of successes, number of trials and probability of success is the number of concordant SNPs, total SNPs and 0.5, respectively migraine $\left(P_{\mathrm{SNP}}=4 \times 10^{-4}\right)$, and was genome-wide significantly associated with $\operatorname{MDD}\left(P_{\mathrm{SNP}}=1.2 \times 10^{-8}\right)$ in the published joint analysis of 23andMe discovery, PGC, and 23andMe replication MDD GWAS [33]. Thus providing an ideal example where combining MDD GWAS and migraine GWAS results can improve power to identify risk loci for MDD. The remaining three SNPs (rs146377178 hg19.chr8: g.25386973C >T, rs672931 hg19.chr11:g.30920897T >C, and rs11858956 hg19.chr15:g.70261228T $>$ C) showed suggestive association with MDD (and are not in LD $\left[r^{2}<\right.$ $0.1]$ with genome-wide significant SNPs) also showed association with migraine $\left(P_{\mathrm{SNP}} \leq 0.005\right)$, and are novel genome-wide significant risk loci. SNP rs146377178 is located between $C D C A 2$ and $E B F 2$ on chromosome 8p21.2, rs672931 is located within $D C D C 5$ on $11 \mathrm{p} 14.1$, and rs 11858956 is located between RPLP1 and TLE3 on $15 \mathrm{q} 23$.

\section{Gene-based genetic overlap between migraine and MDD}

As summarized in Table 4, similar to the SNP-based analysis, a significant gene-level genetic overlap between migraine and MDD was observed. For instance, a significant proportion of genes with gene-based $P$ value $\leq 0.05$ overlapped between migraine and $\operatorname{MDD}\left(P_{\text {binomial-test }}=\right.$ $0.001)$ : the observed proportion $(8.7 \%)$ of genes with a gene-based $P$ value $\leq 0.05$ in both migraine and MDD is significantly higher than the expected proportion $(6.5 \%)$ of genes with $P$ value $\leq 0.05$ for MDD. Furthermore, the use of a less stringent gene-based $P$ value threshold $\leq 0.1$ produced more significant genetic overlap across two disorders
$\left(P_{\text {binomial-test }}=2.60 \times 10^{-6}\right)$, while the use of a more stringent gene-based $P$ value threshold $\leq 0.01$ produced less significant genetic overlap $\left(P_{\text {binomial-test }}=0.045\right)$.

Combining gene-based evidence for association across the disorders (2016 IHGC migraine and 2013 PGC MDD) using Fisher's combined test (Table 5), two genes were identified with combined $P$ values that are below the genome-wide significance threshold adjusted for 13,524 independent gene-based tests $\left(3.7 \times 10^{-6}=0.05 / 13,524\right)$, namely $A N K D D 1 B$ on chromosome $5 \mathrm{q} 13.3$ and $K C N K 5$ on 6p21.1. Interestingly, the effect alleles of the top SNPs driving the ANKDD1B gene-based association (rs34358 hg19.chr5:g.74965122G $>$ A for migraine and rs904743 hg19.chr5:g.74917862A $>\mathrm{G}$ for MDD) and the KCNK5 association (rs9394578 hg19.chr6:g.39165859C $>$ A for migraine and rs2815095 hg19.chr6:g.39156108T $>C$ for MDD) had opposite effects on risk for migraine and MDD, and were in weak LD $\left(r^{2}=0.24\right.$ and 0.39 , respectively), providing evidence for allelic heterogeneity at these associated genes.

\section{Pathway analysis of the overlapping genes}

Pathway analysis was performed for a total of 86 overlapping genes with $P_{\text {Fisher's-combined }} \leq 1 \times 10^{-3}$ (see Supplementary Table 2). After excluding pathways having common genes with top significant SNPs in LD $r^{2}>0.1,39$ pathways with 21 genes were enriched $(F D R \leq 0.05)$ with at least one annotation of a human gene (summarized in Table 6), from which 10 pathways with 12 genes showed at least two human gene annotations. 
Table 3 Summary of the independent genome-wide significant SNPs from meta-analysis of 2016 IHGC migraine and 201623 andMe MDD GWAS

\begin{tabular}{|c|c|c|c|c|c|c|c|c|c|c|c|}
\hline \multirow[t]{2}{*}{ SNP } & \multirow[t]{2}{*}{ Chr } & \multirow[t]{2}{*}{ Position } & \multirow[t]{2}{*}{ RefSeq gene context } & \multirow[t]{2}{*}{ EA } & \multirow[t]{2}{*}{ NEA } & \multicolumn{2}{|c|}{ FE meta-analysis } & \multicolumn{2}{|c|}{ Migraine } & \multicolumn{2}{|c|}{ MDD } \\
\hline & & & & & & OR & $P$ value & OR & $P$ value & OR & $P$ value \\
\hline rs12127789 & 1 & 72740073 & {$[N E G R 1]$} & $\mathrm{T}$ & G & 1.05 & $7.20 \times 10^{-9}$ & 1.04 & 0.015 & 1.05 & $1.67 \times 10^{-7}$ \\
\hline rs2195636 & 3 & 158352440 & $M L F 1-[]-G F M 1$ & $\mathrm{~T}$ & $\mathrm{C}$ & 1.03 & $1.42 \times 10^{-8}$ & 1.02 & 0.048 & 1.04 & $5.63 \times 10^{-8}$ \\
\hline rs768705 & 5 & 87568710 & [TMEM161B-AS1] & A & G & 0.97 & $1.52 \times 10^{-8}$ & 1.01 & 0.22 & 0.95 & $6.58 \times 10^{-13}$ \\
\hline rs146377178 & 8 & 25386973 & $C D C A 2-[]--E B F 2$ & $\mathrm{~T}$ & $\mathrm{C}$ & 1.09 & $7.41 \times 10^{-9}$ & 1.09 & $4.24 \times 10^{-3}$ & 1.09 & $6.13 \times 10^{-7}$ \\
\hline rs6476606 & 9 & 37005561 & [PAX5] & A & G & 1.03 & $2.52 \times 10^{-8}$ & 1.04 & $2.98 \times 10^{-4}$ & 1.03 & $1.50 \times 10^{-5}$ \\
\hline rs672931 & 11 & 30920897 & {$[D C D C 1]$} & $\mathrm{T}$ & $\mathrm{C}$ & 1.03 & $7.01 \times 10^{-9}$ & 1.04 & $8.27 \times 10^{-4}$ & 1.03 & $2.34 \times 10^{-6}$ \\
\hline rs9536359 & 13 & 53691446 & OLFM4--[]-LINC01065 & $\mathrm{T}$ & $\mathrm{C}$ & 0.96 & $2.75 \times 10^{-13}$ & 0.97 & $3.88 \times 10^{-3}$ & 0.96 & $1.15 \times 10^{-11}$ \\
\hline rs11858956 & 15 & 70261228 & RPLP1--[]-TLE3 & $\mathrm{T}$ & $\mathrm{C}$ & 0.97 & $4.20 \times 10^{-8}$ & 0.97 & $3.51 \times 10^{-3}$ & 0.97 & $3.27 \times 10^{-6}$ \\
\hline rs5751069 & 22 & 41627775 & {$[C H A D L]$} & $\mathrm{C}$ & G & 0.96 & $5.42 \times 10^{-11}$ & 0.96 & $4.58 \times 10^{-4}$ & 0.96 & $2.51 \times 10^{-8}$ \\
\hline
\end{tabular}

SNP dbSNP v147 variant ID, Chr chromosome, Position hg19 human genome assembly, RefSeq gene context for genic SNPs, the relevant gene is listed within brackets and for intergenic SNPs the genes flanking the locus are listed, $E A$ effect allele, $N E A$ non-effect allele, $F E$ meta-analysis fixed effect model meta-analysis, OR odds ratio for the EA, Migraine 2016 IHGC migraine GWAS, MDD 2016 23andMe MDD discovery GWAS

Among these pathway terms, neural-related pathways were substantially over-represented, especially for pathways related to signalling (e.g., "Wnt signalling pathway"; KEGG:04310; PLCB3, SERPINF1 and DVL3; FDR $P$ value $=0.02$ ) and the ion channel regulations (e.g., "potassium ion leak channel activity"; GO:0022841; $K C N K 5$ and $K C N K 4$; FDR $P$ value $=0.02$ ), both of which were also strongly dominant in the pathways with at least two human gene annotations. Enzymatic activity-related pathways (e.g., "serine O-acyltransferase activity"; GO:0016412; MBOAT4; FDR $P$ value $=0.05)$ and metabolic pathways (e.g., "arsonoacetate metabolic process"; GO:0018872; AS3MT; FDR $P$ value $=0.05$ ) were also observed to be enriched in migraine and/or MDD aetiology. Notably, only one term "endocrine and other factorregulated calcium reabsorption" (KEGG:04961; PLCB3 and $A P 2 M 1$; Bonferroni corrected $P$ value $=0.05$ ) remained after using more conservative Bonferroni correction for multiple testing, which is related to processes of intracellular signalling and neuronal excitability [53].

Extending pathway analyses to also include functional annotations of GO assigned by in silico curation methods (Inferred from Electronic Annotation [IEA]) provided further evidence for the molecular signalling-related pathways involved in migraine and/or MDD aetiology, with 38 additional pathways (see Supplementary Table 3) represented by 11 genes (ECM1, DLST, TMEM208, PLXNB1, RNF113B, FARP1, CLEC17A, GPR126, CENPH, GRK6, and TFB1M). Importantly, this analysis highlighted sevenpathways with at least two human gene annotations: "regulation of release of cytochrome c from mitochondria" (GO:0090199; NOL3 and $B A D$; FDR $P$ value $=0.03$ ); "negative regulation of peptidase activity" (GO:0010466;NOL3, ECM1, NGF and SERPINF1; FDR $P$ value $=0.03$ ); "negative regulation of cytokine-mediated signalling pathway" (GO:0001960; NOL3 and $E C M 1$; FDR $P$ value $=0.03$ ); "Rac GTPase binding" (GO:0048365; DVL3 and FARPI; FDR $P$ value $=0.03$ ); "cysteine-type endopeptidase regulator activity involved in apoptotic process" (GO:0043028; NOL3 and BAD; FDR $P$ value $=0.03$ ); "extracellular matrix binding" (GO:0050840; $E C M 1$ and GPR126; FDR $P$ value $=0.05$ ); and "death receptor binding" (GO:0005123, NOL3 and NGF; FDR $P$ value $=0.05$ )

\section{Discussion}

Here we performed a comprehensive analysis to assess the genetic overlap between migraine and MDD using three GWAS data sets, which is the first systematic study aimed at identifying shared genetic factors between migraine and MDD at the molecular genetic level. Several interesting findings are noteworthy.

Firstly, we estimated a significant SNP-based liabilityscale heritability of $12 \%$ using the 2016 IHGC migraine GWAS data set and 19\% using the 2013 PGC MDD GWAS data set. The SNP-based heritability estimates of migraine and MDD are lower than those estimated from twin and family studies $[10,11]$. This so-called "missing heritability" is likely due to the combined effects of rare SNPs and SNPs with small effects that are difficult to capture using current GWAS sample sizes and analysis of common SNPs $[54,55]$.

Our study reported a significant SNP-based $r_{\mathrm{G}}$ of 0.25 between migraine (2016 IHGC) and MDD (2013 PGC), which is similar to estimates $\left(r_{\mathrm{G}}=0.30-0.36\right)$ from twin and family studies $[13,14]$. Although the $r_{\mathrm{G}}$ between 2016 IHGC migraine and 2016 23andMe MDD could not be 
Table 4 Summary of genebased association analyses under three $P$ value thresholds

\begin{tabular}{|c|c|c|c|c|c|c|}
\hline \multirow[t]{2}{*}{ Discovery } & \multirow[t]{2}{*}{ Target } & \multicolumn{2}{|c|}{ Overlapping genes } & \multicolumn{2}{|c|}{ Proportion of overlap } & \multirow[t]{2}{*}{ Binominal test $P$ value } \\
\hline & & Raw & Effective & Expected & Observed & \\
\hline \multicolumn{7}{|c|}{$P$ value $\leq 0.01$} \\
\hline $\begin{array}{l}\text { Migraine } \\
P \text { value } \leq 0 .\end{array}$ & MDD & 21 & 12 & 0.016 & 0.027 & 0.045 \\
\hline $\begin{array}{l}\text { Migraine } \\
P \text { value } \leq 0\end{array}$ & MDD & 173 & 115 & 0.065 & 0.087 & 0.001 \\
\hline Migraine & MDD & 468 & 318 & 0.12 & 0.15 & $2.60 \times 10^{-6}$ \\
\hline
\end{tabular}

Migraine 2016 IHGC migraine GWAS, MDD 2013 PGC MDD GWAS, Raw total number of genes, effective effective number of independent genes assessed via LD Score regression (due to the restricted availability of genome-wide results for the 23andMe MDD GWAS), our SECA results provided strong evidence for a significant genetic overlap, indicated by the significant enrichment in concordant SNP effects across the 2016 IHGC migraine and 2016 23andMe MDD GWAS.

Given the strong evidence for shared genetic factors, we performed a meta-analysis of the 2016 IHGC migraine and 2016 23andMe MDD GWA studies. No substantial difference was observed when comparing results of the FE and the RE2 models (Supplementary Table 1), indicating negligible SNP effect heterogeneity across studies. The metaanalysis identified 3 novel (index) SNP loci near several genes: rs146377178 between $C D C A 2$ and $E B F 2$, rs672931 within DCDC5, and rs11858956 between RPLP1 and TLE3. Whereas little evidence exists that supports a biological role in migraine or MDD risk for DCDC5 and TLE3, at least some evidence is reported in the literature for the other three genes. Specifically, $C D C A 2$, which is related to cell division cycle, was previously observed to be involved in the overlapping pathways across migraine with aura and migraine without aura [42]; $E B F 2$ is reported to play a role in regulating dopaminergic neurons in the midbrain periaqueductal grey matter, which is relevant to pain modulation [56], and therefore may contribute to both migraine and MDD risk; and RPLPl was revealed to be related to MDD in a mouse model, suggesting that the ribosome pathways of proteins synthesis/degradation were implicated in MDD aetiology [57].

One SNP (rs6476606) showed association in both the 2016 IHGC migraine GWAS $\left(P_{\mathrm{SNP}}=0.0003\right)$ and 2016 23andMe MDD discovery GWAS $\left(P_{\mathrm{SNP}}=1.50 \times 10^{-5}\right)$, with genome-wide significant evidence for association in the FE meta-analysis $\left(P_{\mathrm{SNP}}=2.52 \times 10^{-8}\right)$, and genomewide significant association with MDD in the joint analysis of 23andMe discovery, PGC, and 23andMe replication MDD GWAS $\left(P_{\mathrm{SNP}}=1.2 \times 10^{-8}\right)$ [33]. This indicates that combining migraine and MDD GWAS data has the potential to identify robust MDD risk loci. Interestingly, this finding is in line with previous results suggesting that in at least a subset of migraine patients with MDD, migraine may be a symptom or consequence of MDD [58]. Further research will be required to determine whether combining migraine and MDD GWAS data can help to identify robust migraine risk loci (e.g., utilizing genome-wide results from more powerful MDD GWAS).

Extending our analysis from SNP-level to gene-level revealed a significant genetic overlap across migraine and MDD, providing additional evidence for such overlap ('pleiotropy') between the disorders. Application of Fisher's combined test identified two genes with genome-wide significant gene-based $P$ values (ANKDDIB and KCNK5). Although minimal data exist for $A N K D D 1 B$, it may be relevant to migraine and MDD susceptibility due to its role in coding ankyrin-repeat proteins, which have been associated with a number of human disorders, and include the Notch protein (a key component of cell signalling pathways) in which mutations can cause cerebral autosomal dominant arteriopathy with subcortical infarcts and leukoencephalopathy (CADASIL) - for which the most common clinical manifestations are migraine headaches and transient ischaemic attacks [59]. In contrast, the two-pore forming potassium channel gene $K C N K 5$ is an attractive candidate for disorders of the central nervous system and other members of this protein family have already been linked to migraine or MDD susceptibility. For instance, although the genetic evidence has been debated [60, 61], the TWIK-related spinal cord potassium channel (TRESK, encoded by $K C N K 18$ ) has been associated with migraine susceptibility [62, 63]; and the inactivation of the TWIKrelated potassium channel (TREK, encoded by $K C N K 4$ ) produces a depression-resistant phenotype in a mouse model [64].

Based on the significant overlapping genes identified in gene-based association analyses, multiple pathways were observed, which were over-presented in neural-related pathways such as metal ion channel regulations, signalling pathways, and enzymatic activity. These results provide evidence for the importance of neurological mechanisms on triggering comorbid migraine and MDD, suggesting that 


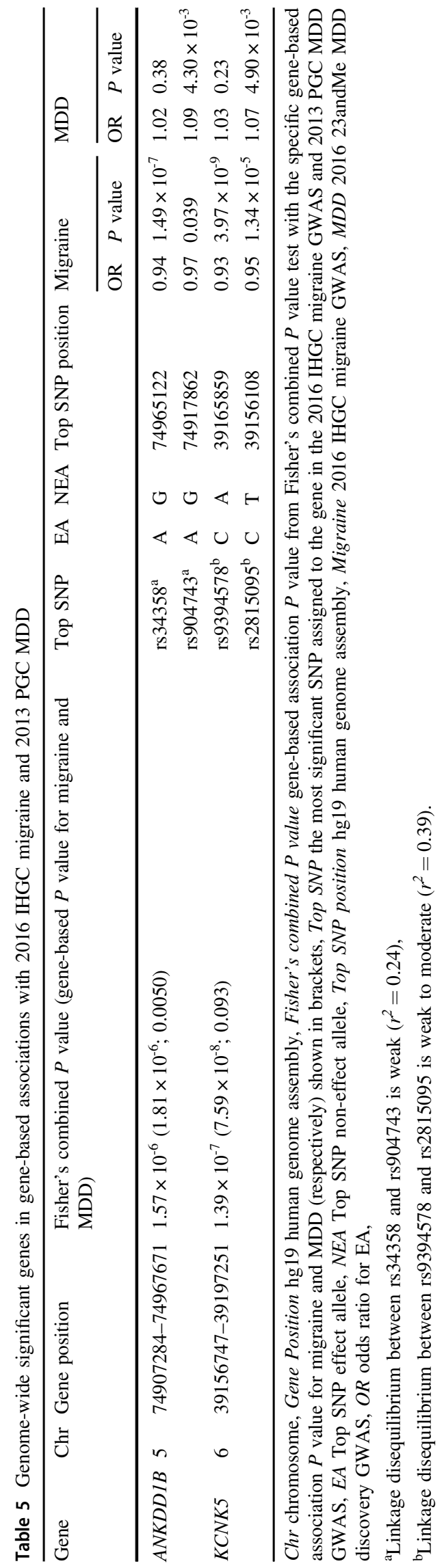

comorbid migraine and MDD may be induced by their shared neurological symptoms.

Our study has limitations. Firstly, LD Score regression defaults with calculating heritability based on the observed scale. Although we converted the conditions onto the liability scale by setting a fixed population and sample prevalence of migraine and MDD, the estimates may still be underestimated due to the relatively high sample prevalence of PGC MDD (around 50\%). In addition, we identified a very small genetic covariance intercept of 0.04 , indicating a small sample overlap between 2016 IHGC migraine GWAS and 2013 PGC MDD GWAS that may have influenced our gene-based association analyses. However, we believe such effects will be negligible since the intercept is very close to zero. Moreover, the restricted availability of genome-wide GWAS summary statistics for the 2016 23andMe MDD GWAS limited our findings: (1) we could not estimate a genetic correlation between the 2016 IHGC migraine and 2016 23andMe MDD GWAS using LD Score regression; hence, we utilized SECA to test for their genetic overlap; (2) it is possible most of the genome-wide significant SNPs from meta-analysis of migraine and MDD showed a stronger signal for MDD compared to migraine because the 201623 andMe MDD GWAS was limited to the top 10,000 SNPs (i.e., 'weaker' MDD SNP associations were not metaanalysed); and (3) we could not perform a complete genome-wide meta-analysis of the 2016 IHGS migraine and 2016 23andMe MDD GWAS; nor could we perform genebased genetic overlap analysis across the 2016 IHGC migraine and 201623 andMe MDD GWAS. Lastly, because our gene-based association tests assigned SNPs to genes based on close proximity (i.e., within $15 \mathrm{~kb} 5^{\prime}$ of the TSS site and $15 \mathrm{~kb} \mathrm{3}$ ' of the TES), more distant SNPs associated with gene-to-phenotype risk may influence the interpretation of our gene-based overlap analyses.

In conclusion, we have shown a significant genetic overlap across migraine and MDD at both the SNP- and gene-level. Importantly, we identified three novel independent genome-wide significant SNPs (rs146377178, rs672931, and rs11858956; located between $C D C A 2$ and $E B F 2$, within DCDC5, and between RPLP1 and TLE3, respectively), and two genome-wide significant genes (ANKDD1B and KCNK5). Multiple pathway terms, especially the neural-related pathways of signalling and metal ion channel regulation, were implicated. Overall, our study provides strong molecular genetic support for shared genetically controlled mechanisms underlying migraine and MDD risk, and provide impetus to perform further combined analyses of migraine and MDD GWAS data.

Acknowledgements $\mathrm{HZ}$ was supported by a National Health and Medical Research Council (NHMRC) Early Career Fellowship (APP1091816). DRN was supported by an NHMRC Research 
Table 6 Summary of independent pathway analysis

\begin{tabular}{|c|c|c|c|}
\hline Pathway term name & Pathway term ID & $\begin{array}{l}\text { FDR-corrected } P \\
\text { value }\end{array}$ & Gene list \\
\hline \multicolumn{4}{|l|}{ Source: Gene Ontology (biological process) } \\
\hline Stabilization of membrane potential & GO:0030322 & 0.021 & KCNK5, KCNK4 \\
\hline Protein octanoylation & GO:0018190 & 0.050 & MBOAT4 \\
\hline Arsonoacetate metabolic process & GO:0018872 & 0.050 & $A S 3 M T$ \\
\hline $\begin{array}{l}\text { Mitochondrial threonyl-tRNA } \\
\text { aminoacylation }\end{array}$ & GO:0070159 & 0.050 & TARS2 \\
\hline \multicolumn{4}{|l|}{ Source: Gene Ontology (cellular component) } \\
\hline $\begin{array}{l}\text { Mitochondrial oxoglutarate dehydrogenase } \\
\text { complex }\end{array}$ & GO:0009353 & 0.050 & MRPS36 \\
\hline \multicolumn{4}{|l|}{ Source: Gene Ontology (molecular function) } \\
\hline Potassium ion leak channel activity & GO:0022841 & 0.021 & KCNK5,KCNK4 \\
\hline Peptidase regulator activity & GO:0061134 & 0.023 & $\begin{array}{l}\text { BAD,SERPINF1, } \\
N G F, N O L 3\end{array}$ \\
\hline Nerve growth factor receptor binding & GO:0005163 & 0.050 & $N G F$ \\
\hline $\begin{array}{l}\text { Extracellular-glutamate-gated chloride } \\
\text { channel activity }\end{array}$ & GO:0008068 & 0.050 & GABRP \\
\hline Serine $O$-acyltransferase activity & GO:0016412 & 0.050 & MBOAT4 \\
\hline Arsenite methyltransferase activity & GO:0030791 & 0.050 & $A S 3 M T$ \\
\hline Methylarsonite methyltransferase activity & GO:0030792 & 0.050 & $A S 3 M T$ \\
\hline Nucleoside phosphotransferase activity & GO:0050146 & 0.050 & NT5C2 \\
\hline $\begin{array}{l}\text { Mechanically gated potassium channel } \\
\text { activity }\end{array}$ & GO:0098782 & 0.050 & KCNK4 \\
\hline \multicolumn{4}{|c|}{ Source: Kyoto Encyclopedia of Genes and Genomes (KEGG) } \\
\hline Wnt signalling pathway & KEGG:04310 & 0.020 & $\begin{array}{l}\text { SERPINF1,PLCB3, } \\
\text { DVL3 }\end{array}$ \\
\hline $\begin{array}{l}\text { Endocrine and other factor-regulated } \\
\text { calcium reabsorption }\end{array}$ & KEGG:04961 & 0.016 & $P L C B 3, A P 2 M 1$ \\
\hline Amyotrophic lateral sclerosis (ALS) & KEGG:05014 & 0.019 & $B A D, N E F M$ \\
\hline \multicolumn{4}{|l|}{ Source: Reactome } \\
\hline G1 phase & REAC:69236 & 0.012 & $C D K 7, E 2 F 4$ \\
\hline Retrograde neurotrophin signalling & REAC:177504 & 0.0017 & $N G F, A P 2 M 1$ \\
\hline Phase 4 -resting membrane potential & REAC:5576886 & 0.0031 & KCNK5,KCNK4 \\
\hline $\begin{array}{l}\text { RNA polymerase II transcribes snRNA } \\
\text { genes }\end{array}$ & REAC:6807505 & 0.050 & $C D K 7, R P R D 2$ \\
\hline $\begin{array}{l}\text { BH3-only proteins associate with and } \\
\text { inactivate anti-apoptotic BCL-2 members }\end{array}$ & REAC: 111453 & 0.048 & $B A D$ \\
\hline $\begin{array}{l}\text { Nef-mediated downregulation of CD28 cell } \\
\text { surface expression }\end{array}$ & REAC:164939 & 0.012 & $A P 2 M 1$ \\
\hline NGF processing & REAC: 167060 & 0.024 & $N G F$ \\
\hline Nef-mediated CD8 downregulation & REAC: 182218 & 0.042 & $A P 2 M 1$ \\
\hline TRKA activation by NGF & REAC: 187042 & 0.012 & $N G F$ \\
\hline NRAGE signals death through JNK & REAC: 193648 & 0.020 & $B A D, N G F$ \\
\hline $\begin{array}{l}\text { p75NTR negatively regulates cell cycle via } \\
\text { SC1 }\end{array}$ & REAC: 193670 & 0.036 & $N G F$ \\
\hline Ceramide signalling & REAC:193681 & 0.018 & $N G F$ \\
\hline Signalling to STAT3 & REAC: 198745 & 0.018 & $N G F$ \\
\hline NFG and proNGF binds to p75NTR & REAC:205017 & 0.018 & $N G F$ \\
\hline Axonal growth stimulation & REAC:209563 & 0.024 & $N G F$ \\
\hline & REAC:434316 & 0.048 & $P L C B 3$ \\
\hline
\end{tabular}


Table 6 (continued)

\begin{tabular}{llll}
\hline Pathway term name & Pathway term ID & $\begin{array}{l}\text { FDR-corrected } P \\
\text { value }\end{array}$ & Gene list
\end{tabular}

Fatty acids bound to GPR40 (FFAR1)

regulate insulin secretion

TWIK-related potassium channel (TREK)

Abacavir metabolism

SUMO is conjugated to E1 (UBA2:SAE1)

SUMO is transferred from E1 to E2

(UBE2I, UBC9)

Regulation by TREX1

$\begin{array}{lll}\text { REAC:1299503 } & 0.018 & \text { KCNK4 } \\ \text { REAC:2161541 } & 0.030 & \text { NT5C2 } \\ \text { REAC:3065676 } & 0.030 & \text { SAE1 } \\ \text { REAC:3065678 } & 0.036 & \text { SAE1 } \\ & & \\ \text { REAC:3248023 } & 0.018 & \text { TREX1 } \\ \text { REAC:5368598 } & 0.030 & \text { DVL3 }\end{array}$

Negative regulation of TCF-dependent signalling by DVL-interacting proteins

$F D R$ false discovery rate

Fellowship (APP0613674). This work was supported by an NHMRC project grant (APP1075175), the European Union's Seventh Framework Program (2007-2013) under grant agreement no. 602633 (EUROHEADPAIN) and the US National Institutes of Health (AA07535, AA011998, AA017688, AA10249, AA13320, AA13321, AA13326, AA14041, MH66206, DA12854, DA019951). We are grateful to Lisanne S. Vijfhuizen (Leiden University Medical Center) for assistance with the revision. We thank the PGC for making their 2013 MDD GWAS summary statistics publicly available.

\section{Compliance with ethical standards}

Conflict of interest The authors declare that they have no conflict of interest.

\section{References}

1. Launer LJ, Terwindt GM, Ferrari MD. The prevalence and characteristics of migraine in a population-based cohort: the GEM study. Neurology. 1999;53:537-542.

2. Bhattarai N, Charlton J, Rudisill C, Gulliford MC. Prevalence of depression and utilization of health care in single and multiple morbidity: a population-based cohort study. Psychol Med. 2013;43:1423-1431.

3. Kessler RC, Berglund P, Demler O, et al. The epidemiology of major depressive disorder: results from the National Comorbidity Survey Replication (NCS-R). JAMA. 2003;289:30953105.

4. Breslau N, Davis GC, Schultz LR, Peterson EL. Joint 1994 Wolff Award Presentation. Migraine and major depression: a longitudinal study. Headache. 1994;34:387-393.

5. Breslau N, Lipton RB, Stewart WF, Schultz LR, Welch KM. Comorbidity of migraine and depression: investigating potential etiology and prognosis. Neurology. 2003;60:1308-1312.

6. Breslau N, Schultz LR, Stewart WF, Lipton RB, Lucia VC, Welch $\mathrm{KM}$. Headache and major depression: is the association specific to migraine? Neurology. 2000;54:308-313.

7. Modgill G, Jette N, Wang JL, Becker WJ, Patten SB. A population-based longitudinal community study of major depression and migraine. Headache. 2012;52:422-432.

8. Mongini F, Keller R, Deregibus A, Raviola F, Mongini T, Sancarlo M. Personality traits, depression and migraine in women: a longitudinal study. Cephalalgia. 2003;23:186-192.

9. Vos T, Flaxman AD, Naghavi M, et al. Years lived with disability (YLDs) for 1160 sequelae of 289 diseases and injuries 1990-2010: a systematic analysis for the Global Burden of Disease Study 2010. Lancet. 2012;380:2163-2196.

10. Mulder EJ, Van Baal C, Gaist D, et al. Genetic and environmental influences on migraine: a twin study across six countries. Twin Res. 2003;6:422-431.

11. Bierut LJ, Heath AC, Bucholz KK, et al. Major depressive disorder in a community-based twin sample: are there different genetic and environmental contributions for men and women? Arch Gen Psychiatry. 1999;56:557-563.

12. Stam AH, de Vries B, Janssens AC, et al. Shared genetic factors in migraine and depression: evidence from a genetic isolate. Neurology. 2010;74:288-294.

13. Schur EA, Noonan C, Buchwald D, Goldberg J, Afari N. A twin study of depression and migraine: evidence for a shared genetic vulnerability. Headache. 2009;49:1493-1502.

14. Ligthart L, Nyholt DR, Penninx BW, Boomsma DI. The shared genetics of migraine and anxious depression. Headache. 2010;50:1549-1560.

15. Anttila V, Stefansson H, Kallela M, et al. Genome-wide association study of migraine implicates a common susceptibility variant on 8q22.1. Nat Genet. 2010;42:869-873.

16. Anttila V, Winsvold BS, Gormley P, et al. Genome-wide metaanalysis identifies new susceptibility loci for migraine. Nat Genet. 2013;45:912-917.

17. Chasman DI, Schurks M, Anttila V, et al. Genome-wide association study reveals three susceptibility loci for common migraine in the general population. Nat Genet. 2011;43:695-698.

18. Cox HC, Lea RA, Bellis C, et al. A genome-wide analysis of 'Bounty' descendants implicates several novel variants in migraine susceptibility. Neurogenetics. 2012;13:261-266.

19. Freilinger T, Anttila V, de Vries B, et al. Genome-wide association analysis identifies susceptibility loci for migraine without aura. Nat Genet. 2012;44:777-782.

20. Ligthart L, de Vries B, Smith AV, et al. Meta-analysis of genomewide association for migraine in six population-based European cohorts. Eur J Hum Genet. 2011;19:901-907.

21. Gormley P, Anttila V, Winsvold BS, et al. Meta-analysis of 375,000 individuals identifies 38 susceptibility loci for migraine. Nat Genet. 2016;48:856-866.

22. Lewis CM, Ng MY, Butler AW, et al. Genome-wide association study of major recurrent depression in the U.K. population. Am J Psychiatry. 2010;167:949-957.

23. Major Depressive Disorder Working Group of the Psychiatric GWAS Consortium, Ripke S, Wray NR, et al. A mega-analysis of genome-wide association studies for major depressive disorder. Mol Psychiatry. 2013;18:497-511. 
24. Muglia P, Tozzi F, Galwey NW, et al. Genome-wide association study of recurrent major depressive disorder in two European case-control cohorts. Mol Psychiatry. 2010;15:589-601.

25. Power RA, Cohen-Woods S, Ng MY, et al. Genome-wide association analysis accounting for environmental factors through propensity-score matching: application to stressful live events in major depressive disorder. Am J Med Genet B Neuropsychiatr Genet. 2013;162B:521-529.

26. Rietschel M, Mattheisen M, Frank J, et al. Genome-wide association-, replication-, and neuroimaging study implicates HOMER1 in the etiology of major depression. Biol Psychiatry. 2010;68:578-585.

27. Shi J, Potash JB, Knowles JA, et al. Genome-wide association study of recurrent early-onset major depressive disorder. Mol Psychiatry. 2011;16:193-201.

28. Sullivan PF, de Geus EJ, Willemsen G, et al. Genome-wide association for major depressive disorder: a possible role for the presynaptic protein piccolo. Mol Psychiatry. 2009;14:359-375.

29. Wray NR, Pergadia ML, Blackwood DH, et al. Genome-wide association study of major depressive disorder: new results, metaanalysis, and lessons learned. Mol Psychiatry. 2012;17:36-48.

30. Aragam N, Wang KS, Pan Y. Genome-wide association analysis of gender differences in major depressive disorder in the Netherlands NESDA and NTR population-based samples. J Affect Disord. 2011;133:516-521.

31. Kohli MA, Lucae S, Saemann PG, et al. The neuronal transporter gene SLC6A15 confers risk to major depression. Neuron. 2011;70:252-265.

32. CONVERGE consortium. Sparse whole-genome sequencing identifies two loci for major depressive disorder. Nature. 2015;523:588-591.

33. Hyde CL, Nagle MW, Tian C, et al. Identification of 15 genetic loci associated with risk of major depression in individuals of European descent. Nat Genet. 2016;48:1031-1036.

34. Headache Classification Committee of the International Headache Society (IHS). The International Classification of Headache Disorders, 3rd edition (beta version). Cephalalgia. 2013; 33:629-808.

35. 1000 Genomes Project Consortium, Abecasis GR, Auton A, et al. An integrated map of genetic variation from 1,092 human genomes. Nature. 2012;491:56-65.

36. Magi R, Morris AP. GWAMA: software for genome-wide association meta-analysis. BMC Bioinform. 2010;11:288.

37. American Psychiatric Association. Diagnostic and statistical manual of mental disorders. 4th ed. Washington, DC: American Psychiatric Association; 1994.

38. Bulik-Sullivan BK, Loh PR, Finucane HK, et al. LD Score regression distinguishes confounding from polygenicity in genome-wide association studies. Nat Genet. 2015;47:291-295.

39. Nyholt DR. SECA: SNP effect concordance analysis using genome-wide association summary results. Bioinformatics. 2014:30:2086-2088.

40. Han B, Eskin E. Random-effects model aimed at discovering associations in meta-analysis of genome-wide association studies. Am J Hum Genet. 2011;88:586-598.

41. Purcell S, Neale B, Todd-Brown K, et al. PLINK: a tool set for whole-genome association and population-based linkage analyses. Am J Hum Genet. 2007;81:559-575.

42. Zhao H, Eising E, de Vries B, et al. Gene-based pleiotropy across migraine with aura and migraine without aura patient groups. Cephalalgia. 2016;36:648-657.

43. Pickrell JK, Marioni JC, Pai AA, et al. Understanding mechanisms underlying human gene expression variation with RNA sequencing. Nature. 2010;464:768-772.
44. Li MX, Gui HS, Kwan JS, Sham PC. GATES: a rapid and powerful gene-based association test using extended Simes procedure. Am J Hum Genet. 2011;88:283-293.

45. Chanda P, Huang H, Arking DE, Bader JS. Fast association tests for genes with FAST. PLoS ONE. 2013;8:e68585.

46. Li MX, Yeung JM, Cherny SS, Sham PC. Evaluating the effective numbers of independent tests and significant p-value thresholds in commercial genotyping arrays and public imputation reference datasets. Hum Genet. 2012;131:747-756.

47. Zhao H, Nyholt DR. Gene-based analyses reveal novel genetic overlap and allelic heterogeneity across five major psychiatric disorders. Hum Genet. 2017;136:263-274.

48. Reimand J, Arak T, Adler P, et al. g:Profiler-a web server for functional interpretation of gene lists (2016 update). Nucleic Acids Res. 2016;44:W83-89.

49. Cross-Disorder Group of the Psychiatric Genomics Consortium. Identification of risk loci with shared effects on five major psychiatric disorders: a genome-wide analysis. Lancet. 2013;381:1371-1379.

50. Ashburner M, Ball CA, Blake JA, et al. Gene ontology: tool for the unification of biology. The Gene Ontology Consortium. Nat Genet. 2000;25:25-29.

51. Kanehisa M, Goto S. KEGG: kyoto encyclopedia of genes and genomes. Nucleic Acids Res. 2000;28:27-30.

52. Croft D, Mundo AF, Haw R, et al. The Reactome pathway knowledgebase. Nucleic Acids Res. 2014;42:D472-477.

53. Boros S, Bindels RJ, Hoenderop JG. Active $\mathrm{Ca}(2+)$ reabsorption in the connecting tubule. Pflug Arch. 2009;458:99-109.

54. Lee SH, Wray NR, Goddard ME, Visscher PM. Estimating missing heritability for disease from genome-wide association studies. Am J Hum Genet. 2011;88:294-305.

55. Manolio TA, Collins FS, Cox NJ, et al. Finding the missing heritability of complex diseases. Nature. 2009;461:747-753.

56. Zhang, P., Xia, N., Reijo Pera, R.A. Directed Dopaminergic Neuron Differentiation from Human Pluripotent Stem Cells. $J$. Vis. Exp. (91), e51737, doi:10.3791/51737 (2014)

57. Zubenko GS, Hughes HB 3rd, Jordan RM, Lyons-Weiler J, Cohen BM. Differential hippocampal gene expression and pathway analysis in an etiology-based mouse model of major depressive disorder. Am J Med Genet B Neuropsychiatr Genet. 2014;165B:457-466.

58. Ligthart L, Hottenga JJ, Lewis CM, et al. Genetic risk score analysis indicates migraine with and without comorbid depression are genetically different disorders. Hum Genet. 2014;133:173-186.

59. Mosavi LK, Cammett TJ, Desrosiers DC, Peng ZY. The ankyrin repeat as molecular architecture for protein recognition. Protein Sci. 2004;13:1435-1448.

60. Eising E, de Vries B, Ferrari MD, Terwindt GM, van den Maagdenberg AM. Pearls and pitfalls in genetic studies of migraine. Cephalalgia. 2013;33:614-625.

61. de Vries B, Anttila V, Freilinger T, et al. Systematic re-evaluation of genes from candidate gene association studies in migraine using a large genome-wide association data set. Cephalalgia. 2016;36:604-614.

62. Lafreniere RG, Cader MZ, Poulin JF, et al. A dominant-negative mutation in the TRESK potassium channel is linked to familial migraine with aura. Nat Med. 2010;16:1157-1160.

63. Liu P, Xiao Z, Ren F, et al. Functional analysis of a migraineassociated TRESK $\mathrm{K}+$ channel mutation. $\mathrm{J}$ Neurosci. 2013;33:12810-12824.

64. Thummler S, Duprat F, Lazdunski M. Antipsychotics inhibit TREK but not TRAAK channels. Biochem Biophys Res Commun. 2007;354:284-289. 


\section{Affiliations}

Yuanhao Yang ${ }^{1,2} \cdot$ Huiying Zhao ${ }^{1,3} \cdot$ Dorret I Boomsma $^{4}$ - Lannie Ligthart ${ }^{4}$. Andrea C. Belin ${ }^{5} \cdot$ George Davey Smith $^{6}$. Tonu Esko ${ }^{7,8,9} \cdot$ Tobias M. Freilinger $^{10,11} \cdot$ Thomas Folkmann Hansen $\left(10^{12} \cdot\right.$ M. Arfan Ikram (10) ${ }^{13} \cdot$ Mikko Kallela $^{14}$. Christian Kubisch ${ }^{15}$. Christofidou Paraskevi ${ }^{16}$. David P. Strachan $\left(10^{17} \cdot\right.$ Maija Wessman ${ }^{18,19}$ The International Headache Genetics Consortium • Arn M. J. M. van den Maagdenberg ${ }^{20,21} \cdot$ Gisela M. Terwindt $^{20} \cdot$ Dale R. Nyholt $^{1}$

1 Statistical and Genomic Epidemiology Laboratory, Institute of Health and Biomedical Innovation, Queensland University of Technology, Brisbane, QLD, Australia

2 Institute of Molecular Bioscience, The University of Queensland, Brisbane, QLD, Australia

3 Guangdong Provincial Key Laboratory of Malignant Tumor Epigenetics and Gene Regulation, Sun Yat-sen Memorial Hospital, Sun Yat-sen University, Guangzhou, People's Republic of China

4 Department of Biological Psychology, Vrije Universiteit, Amsterdam, The Netherlands

5 Department of Neuroscience, Karolinska Institutet, Stockholm, Sweden

6 Medical Research Council (MRC) Integrative Epidemiology Unit, University of Bristol, Bristol, UK

7 Medical and Population Genetics Program, Broad Institute of MIT and Harvard, Cambridge, MA, USA

8 Estonian Genome Center, University of Tartu, Tartu, Estonia

9 Division of Endocrinology, Boston Children's Hospital, Boston, MA, USA

10 Department of Neurology and Epileptology, Hertie-Institute for Clinical Brain Research, University of Tübingen,

Tübingen, Germany

11 Institute for Stroke and Dementia Research, Klinikum der
Universität München, Ludwig-Maximilians-Universität München, Munich, Germany

12 Danish Headache Center, Department of Neurology, Rigshospitalet, Glostrup Hospital, University of Copenhagen, Copenhagen, Denmark

13 Department of Epidemiology, Erasmus University Medical Center, Rotterdam, The Netherlands

14 Department of Neurology, Helsinki University Central Hospital, Helsinki, Finland

15 Institute of Human Genetics, University Medical Center HamburgEppendorf, Hamburg, Germany

16 Department of Twin Research and Genetic Epidemiology, King's College London, London, UK

17 Population Health Research Institute, St George's, University of London, London, UK

18 Institute for Molecular Medicine Finland (FIMM), University of Helsinki, Helsinki, Finland

19 Folkhälsan Institute of Genetics, Helsinki, Finland

20 Department of Neurology, Leiden University Medical Center, Leiden, The Netherlands

21 Department of Human Genetics, Leiden University Medical Center, Leiden, The Netherlands

The International Headache Genetics Consortium. Padhraig Gormley 7,22,23,24 • Verneri Anttila7,23,24 . Bendik S. Winsvold ${ }^{25,26,27}$ - Priit Palta ${ }^{18}$ - Tonu Esko ${ }^{7,8,9}$ - Tune H. Pers ${ }^{7,9,28,29} \cdot$ Kai-How Farh ${ }^{7,30,31}$. Ester Cuenca-Leon ${ }^{7,22,23,32} \cdot$ Mikko Muona ${ }^{18,19,33,34} \cdot$ Nicholas A. Furlotte $^{35} \cdot$ Tobias Kurth $^{36,37}$ - Andres Ingason ${ }^{38}$. George McMahon $^{6} \cdot$ Lannie Ligthart $^{4} \cdot$ Gisela M. Terwindt $^{20} \cdot$ Mikko Kallela $^{39} \cdot$ Tobias M. Freilinger $^{10,11}$. Caroline $\operatorname{Ran}^{5}$. Scott G. Gordon ${ }^{40}$. Anine H. Stam ${ }^{20}$. Stacy Steinberg ${ }^{38}$. Guntram Borck ${ }^{41}$ - Markku Koiranen ${ }^{42}$. Lydia Quaye $^{16}$ - Hieab H. H. Adams ${ }^{13,43}$. Terho Lehtimäki ${ }^{44}$. Antti-Pekka Sarin ${ }^{18}$. Juho Wedenoja ${ }^{45}$. David A. Hinds ${ }^{35}$. Julie E. Buring ${ }^{37,46}$ - Markus Schürks ${ }^{47}$ - Paul M. Ridker ${ }^{37,46}$ • Maria Gudlaug Hrafnsdottir ${ }^{48}$. Hreinn Stefansson ${ }^{38}$. Susan M. Ring ${ }^{6}$. Jouke-Jan Hottenga ${ }^{4}$ - Brenda W. J. H. Penninx ${ }^{49} \cdot$ Markus Färkkilä $^{39}$. Ville Artto ${ }^{39}$ - Mari Kaunisto ${ }^{18}$. Salli Vepsäläinen ${ }^{39}$ - Rainer Malik ${ }^{11}$ - Andrew C. Heath ${ }^{50}$ - Pamela A. F. Madden ${ }^{50}$. Nicholas G. Martin ${ }^{40} \cdot$ Grant W Montgomery $^{40} \cdot$ Mitja I Kurki $^{7,18,22,23,51} \cdot$ Mart Kals $^{8} \cdot$ Reedik Mägi $^{8} \cdot$ Kalle Pärn $^{8}$. Eija Hämäläinen ${ }^{18}$ - Hailiang Huang ${ }^{7,23,30}$ - Andrea E. Byrnes ${ }^{7,23,30} \cdot$ Lude Franke $^{52}$ • Jie Huang ${ }^{24}$ - Evie Stergiakouli ${ }^{6}$. Phil H. Lee ${ }^{7,22,23} \cdot$ Cynthia Sandor $^{53} \cdot$ Caleb Webber $^{53} \cdot$ Zameel Cader $^{54,55} \cdot$ Bertram Muller-Myhsok $^{21,56,85}$. Stefan Schreiber ${ }^{57}$. Thomas Meitinger ${ }^{58,59}$ - Johan G. Eriksson ${ }^{60,61} \cdot$ Veikko Salomaa $^{61} \cdot$ Kauko Heikkilä $^{62}$.

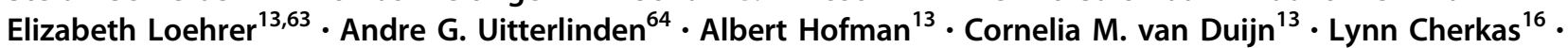
Linda M. Pedersen ${ }^{25}$. Audun Stubhaug ${ }^{65,66} \cdot$ Christopher S. Nielsen ${ }^{65,67} \cdot$ Minna Männikkö $^{42} \cdot$ Evelin Mihailov $^{8}$. Lili Milani ${ }^{8} \cdot$ Hartmut Göbel $^{68} \cdot$ Ann-Louise Esserlind $^{69}$ - Anne Francke Christensen ${ }^{69}$. Thomas Folkmann Hansen ${ }^{70}$. Thomas Werge ${ }^{71,72,73} \cdot$ Jaakko Kaprio ${ }^{18,45,74}$ - Arpo J. Aromaa ${ }^{61}$. Olli Raitakari ${ }^{75,76}$ - M. Arfan Ikram ${ }^{13,43,77}$. 
Tim Spector ${ }^{16} \cdot$ Marjo-Riitta Järvelin ${ }^{42,78,79,80} \cdot$ Andres Metspalu $^{8} \cdot$ Christian Kubisch $^{15} \cdot$ David P. Strachan $^{17}$. Michel D. Ferrari ${ }^{20} \cdot$ Andrea C. Belin $^{5} \cdot$ Martin Dichgans ${ }^{11,81} \cdot$ Maija Wessman $^{18,54}$.

Arn M. J. M. van den Maagdenberg ${ }^{20,21}$ • John-Anker Zwart $25,26,27$. Dorret I. Boomsma ${ }^{4} \cdot$ George Davey Smith ${ }^{6}$. Kari Stefansson ${ }^{38,82} \cdot$ Nicholas Eriksson $^{35} \cdot$ Mark J. Daly $^{7,23,30} \cdot$ Benjamin M. Neale $^{7,23,30} \cdot$ Jes Olesen $^{69}$. Daniel I. Chasman ${ }^{37,46}$ - Dale R. Nyholt ${ }^{83}$ - Aarno Palotie $7,18,22,23,24,30,84$

22 Psychiatric and Neurodevelopmental Genetics Unit, Massachusetts General Hospital and Harvard Medical School, Boston, MA, USA

23 Stanley Center for Psychiatric Research, Broad Institute of MIT and Harvard, Cambridge, MA, USA

24 Wellcome Trust Sanger Institute, Wellcome Trust Genome Campus, Hinxton, UK

25 FORMI, Oslo University Hospital, Oslo, Norway

26 Department of Neurology, Oslo University Hospital, Oslo, Norway

27 Institute of Clinical Medicine, University of Oslo, Oslo, Norway

Department of Epidemiology Research, Statens Serum Institut, Copenhagen, Denmark

29 Novo Nordisk Foundation Center for Basic Metabolic Research, University of Copenhagen, Copenhagen, Denmark

30 Analytic and Translational Genetics Unit, Massachusetts General Hospital and Harvard Medical School, Boston, MA, USA

31 Illumina, San Diego, CA, USA

32 Pediatric Neurology, Vall d'Hebron Research Institute, Barcelona, Spain

33 Neuroscience Center, University of Helsinki, Helsinki, Finland

34 Molecular Neurology Research Program, Research Programs Unit, University of Helsinki, Helsinki, Finland

35 23andMe, Inc., Mountain View, CA, USA

36 Institute of Public Health, Charité-Universitätsmedizin Berlin, Berlin, Germany

37 Division of Preventive Medicine, Brigham and Women's Hospital, Boston, MA, USA

38 deCODE Genetics, Reykjavik, Iceland

39 Department of Neurology, Helsinki University Central Hospital, Helsinki, Finland

40 Department of Genetics and Computational Biology, QIMR Berghofer Medical Research Institute, Brisbane, Queensland, Australia

41 Institute of Human Genetics, Ulm University, Ulm, Germany

42 Center for Life Course Epidemiology and Systems Medicine, University of Oulu, Oulu, Finland

43 Department of Radiology, Erasmus University Medical Center, Rotterdam, The Netherlands

44 Department of Clinical Chemistry, Fimlab Laboratories, School of Medicine, University of Tampere, Tampere, Finland
45 Department of Public Health, University of Helsinki, Helsinki, Finland

46 Harvard Medical School, Boston, MA, USA

47 Department of Neurology, University Duisburg-Essen, Essen, Germany

48 Landspitali University Hospital, Reykjavik, Iceland

49 Department of Psychiatry, VU University Medical Center, Amsterdam, The Netherlands

50 Department of Psychiatry, Washington University School of Medicine, St. Louis, MO, USA

51 Department of Neurosurgery, NeuroCenter, Kuopio University Hospital, Kuopio, Finland

52 Department of Genetics, University Medical Center Groningen, University of Groningen, Groningen, The Netherlands

53 MRC Functional Genomics Unit, Department of Physiology, Anatomy and Genetics, Oxford University, Oxford, UK

54 Nuffield Department of Clinical Neuroscience, University of Oxford, Oxford, UK

55 Oxford Headache Centre, John Radcliffe Hospital, Oxford, UK

56 Max Planck Institute of Psychiatry, Munich, Germany

57 Institute of Clinical Molecular Biology, Christian Albrechts University, Kiel, Germany

58 Institute of Human Genetics, Helmholtz Zentrum München, Neuherberg, Germany

59 Institute of Human Genetics, Technische Universität München, Munich, Germany

60 Department of General Practice and Primary Health Care, University of Helsinki and Helsinki University Hospital, Helsinki, Finland

61 National Institute for Health and Welfare, Helsinki, Finland

62 Institute of Clinical Medicine, University of Helsinki, Helsinki, Finland

63 Department of Environmental Health, Harvard T.H. Chan School of Public Health, Boston, MA, USA

64 Department of Internal Medicine, Erasmus University Medical Center, Rotterdam, The Netherlands

65 Department of Pain Management and Research, Oslo University Hospital, Oslo, Norway

66 Medical Faculty, University of Oslo, Oslo, Norway

67 Department of Ageing and Health, Norwegian Institute of Public Health, Oslo, Norway 
68 Kiel Pain and Headache Center, Kiel, Germany

69 Danish Headache Center, Department of Neurology, Rigshospitalet, Glostrup Hospital, University of Copenhagen, Copenhagen, Denmark

70 Institute of Biological Psychiatry, Mental Health Center Sct. Hans, University of Copenhagen, Roskilde, Denmark

71 Institute of Biological Psychiatry, MHC Sct. Hans, Mental Health Services Copenhagen, Copenhagen, Denmark

72 Institute of Clinical Sciences, Faculty of Medicine and Health Sciences, University of Copenhagen, Copenhagen, Denmark

73 iPSYCH-The Lundbeck Foundation Initiative for Integrative Psychiatric Research, Copenhagen, Denmark

74 Department of Health, National Institute for Health and Welfare, Helsinki, Finland

75 Research Center of Applied and Preventive Cardiovascular Medicine, University of Turku, Turku, Finland

76 Department of Clinical Physiology and Nuclear Medicine, Turku University Hospital, Turku, Finland
77 Department of Neurology, Erasmus University Medical Center, Rotterdam, The Netherlands

78 Department of Epidemiology and Biostatistics, MRC Health Protection Agency (HPE) Centre for Environment and Health, School of Public Health, Imperial College London, London, UK

79 Biocenter Oulu, University of Oulu, Oulu, Finland

80 Unit of Primary Care, Oulu University Hospital, Oulu, Finland

81 Munich Cluster for Systems Neurology (SyNergy), Munich, Germany

82 Faculty of Medicine, University of Iceland, Reykjavik, Iceland

83 Statistical and Genomic Epidemiology Laboratory, Institute of Health and Biomedical Innovation, Queensland University of Technology, Kelvin Grove, Brisbane, QLD, Australia

84 Department of Neurology, Massachusetts General Hospital, Boston, MA, USA

85 Institute of Translational Medicine, University of Liverpool, Liverpool, UK 